\title{
Effect of Acarbose, an Alpha-Glucosidase Inhibitor, on Serum Lipoprotein Lipase Mass Levels and Common Carotid Artery Intima-Media Thickness in Type 2 Diabetes Mellitus Treated by Sulfonylurea
}

Tomokazu Oyama, Atsuhito Saiki, Kei Endoh, Noriko Ban, Daizi, Nagayama, Masahiro Ohhira, Nobukiyo Koide, Yoh Miyashita, and Kohji Shirai

Department of Internal Medicine, Sakura Medical Center, School of Medicine, Toho University, Chiba, Japan.

\begin{abstract}
Aim: Previous reports indicate that serum lipoprotein lipase mass levels (LPL mass) and common carotid artery intima-media thickness (CCA-IMT) are independent predictors of atherosclerotic diseases. The aim of this study was to examine the effects of combination therapy of sulfonylurea and acarbose on LPL mass and CCA-IMT.

Methods: Eighty-four patients with type 2 diabetes mellitus, who were treated with only sulfonylureas and showed CCA-IMT of more than $0.9 \mathrm{~mm}$ at baseline, were selected and randomly divided into two groups. One group was administered acarbose $300 \mathrm{mg} /$ day for $12 \mathrm{months}$ (acarbose group, $n=41$ ), and the other group was not administered acarbose (non-acarbose group, $n=43$ ).

Results: After 12 months, a significant increase in LPL mass and a significant decrease in CCA-IMT were observed in the acarbose group $(1.024$ to $0.964 \mathrm{~mm})$, but no significant changes were observed in the non-acarbose group. In a subgroup analysis of patients with HbA1c improved by $0.5 \%$ or more, the increase of LPL mass and decrease of CCA-IMT was significantly greater in the acarbose group than in the non-acarbose group although the changes in HbAlc were similar in two groups.

Conclusions: We concluded that reducing postprandial hyperglycemia might increase LPL mass levels and might be useful to prevent macroangiopathy in type 2 diabetic patients treated by sulfonylurea.
\end{abstract}

J Atheroscler Thromb, 2008; 15:154-159.

Key words; Type 2 diabetes mellitus, CCA-IMT, LPL mass, Postprandial hyperglycemia, Acarbose

\section{Introduction}

The advent of high-resolution B-mode ultrasonography has allowed noninvasive observation of atherosclerosis in the carotid arteries. Common carotid artery (CCA) wall thickness is evaluated by measuring its intima-media thickness (IMT). CCA-IMT has been reported to be an independent predictor of coronary heart disease and stroke ${ }^{1,2)}$. Recent reports have shown that postprandial hyperglycemia is associated with in-

Address for correspondence: Yoh Miyashita, Department of Internal Medicine, Sakura Medical Center, School of Medicine, Toho University, 564-1 Shimoshizu, Sakura-City, Chiba, 2850841, Japan.

E-mail: mumon@sf6.so-net.ne.jp

Received: October 4, 2007

Accepted for publication: February 2, 2008 creased CCA-IMT ${ }^{3,4)}$ and a higher prevalence of atherosclerotic plaques in the $\mathrm{CCA}^{4,5}$.

Acarbose is an alpha-glucosidase inhibitor that specifically reduces postprandial glucose excursion by delaying the release of glucose from disaccharides and complex carbohydrates in the upper part of the small intestine ${ }^{6}$. Recently, acarbose administration has been shown to delay the progression of IMT in subjects with impaired glucose tolerance ${ }^{3)}$.

Lipoprotein lipase (LPL), which catalyzes the hydrolysis of triglycerides in blood, exists on the endothelial surface and is released into the blood by heparin injection. Analysis of LPL function is important to elucidate lipid metabolism; however, there is almost no LPL activity in the serum under physiological conditions, because LPL remains on the surface of endothelial cells and expresses enzyme activity on the vas- 
cular surface. Therefore, in clinical studies, LPL has been analyzed using serum obtained after intravenous injection of heparin (postheparin LPL), since LPL is detached from the endothelial surface and released in to blood after heparin injection. A considerable level of LPL activity can be observed in serum after intravenous injection of heparin. Postheparin LPL is considered to reflect the amount of LPL production in the whole body.

Recently, a sensitive immunoassay system using specific monoclonal antibody against LPL demonstrated the presence of LPL protein in serum without intravenous injection of heparin (LPL mass) ${ }^{7,8)}$. Tornvall et al. have indicated that LPL mass might be LPL protein detached from the endothelial surface into blood after degradation ${ }^{8}$. We have previously reported that LPL mass was significantly lower in patients with type 2 diabetes mellitus compared to healthy subjects 9,10$)$ and that low serum levels of LPL mass might be a high-risk factor of coronary atherosclerosis ${ }^{11,12}$. These results suggest that LPL mass may be useful as a predictor of atherosclerosis.

We hypothesized that acarbose administration could increase LPL mass and show an anti-atherosclerotic effect in type 2 diabetes mellitus. We therefore examined the effect of combination therapy of sulfonylurea and acarbose on CCA-IMT and LPL mass in type 2 diabetes mellitus.

\section{Methods}

\section{Subjects}

A randomized, open study was performed. Eightyfour patients with type 2 diabetes mellitus, who attended Sakura Medical Center of Toho University as outpatients, were enrolled. All subjects were treated by only sulfonylureas (glibenclamide) as oral hypoglycemic agents and had IMT thickness above $0.9 \mathrm{~mm}$ at baseline, and were divided into two groups by simple randomization using a closed envelope. One group was administered acarbose $300 \mathrm{mg} /$ day for 12 months (acarbose group, $n=41$ ), and the other group was not administered acarbose (non-acarbose group, $n=43$ ). The clinical profile of the subjects is shown in Table $\mathbf{1}$. There were no differences in age, morbidity duration of diabetes mellitus and metabolic parameters between the two groups. There were also no differences in the ratio of subjects using lipid-lowering or blood pressure-lowering drugs between the two groups. All subjects had no previous cardiovascular and cerebrovascular diseases. Subjects were excluded if they received insulin therapy or had diabetic retinopathy and nephropathy. During this study, all subjects maintained
Table 1. Baseline data of two groups

\begin{tabular}{lcc}
\hline & Non-acarbose group & Acarbose group \\
\hline $\mathrm{n}$ (male/female) & $43(18 / 25)$ & $41(12 / 29)$ \\
Age (years) & $63 \pm 4$ & $65 \pm 6$ \\
Morbidity duration of & $11.3 \pm 5.2$ & $11.7 \pm 6.5$ \\
diabetes (years) & $23.1 \pm 3.2$ & $23.4 \pm 2.5$ \\
BMI & $132 \pm 13 / 77 \pm 10$ & $130 \pm 13 / 76 \pm 9$ \\
BP (systolic/diastolic) & $134 \pm 15$ & $141 \pm 19$ \\
FBS (mg/dL) & $4.1 \pm 1.2$ & $3.7 \pm 1.5$ \\
Basal IRI ( $\mu$ U/mL) & $7.4 \pm 0.7$ & $7.5 \pm 0.8$ \\
HbA1c (\%) & $198 \pm 36$ & $198 \pm 25$ \\
TC (mg/dL) & $133 \pm 71$ & $135 \pm 68$ \\
TG (mg/dL) & $52 \pm 14$ & $53 \pm 16$ \\
HDL-C (mg/dL) & $114 \pm 26$ & $116 \pm 34$ \\
LDL-C (mg/dL) & $10(20 \%)$ & $7(18 \%)$ \\
Lipid-lowering drugs & & \\
(No) (all statins) & $7(14 \%)$ & $4(10 \%)$ \\
Blood pressure-lowering & & \\
drugs (No) (all ARBs) & &
\end{tabular}

Data are presented as the mean \pm SD. BMI, body mass index; $\mathrm{BP}$, blood pressure; FBS, fasting bloodglucose; Basal IRI, basal immunoreactiveinsulin; $\mathrm{HbA1c}$, glycosylated hemoglobin; TC, total cholesterol; TG, triglycerides; HDL-C, HDL-cholesterol; LDL-C, LDLcholesterol; CCA-IMT, common carotid artery-intimamedia thickness; statin, HMG-CoAreductaseinhibitor; ARB, angiotensin II receptor antagonist

appropriate diet and exercise therapies, and did not change medications. All subjects received nutrition education from a dietitian every month. The dietitian analyzed a typical meal of the patient and corrected any shortcomings. Exercise therapy was checked by the physician every month. From these check systems, they were confirmed to be following appropriate diet and exercise therapy. This study was approved by the Ethics Committee of Toho University. The purpose and potential risks of this study were explained to all patients and their voluntary written consent for participation in the study and also for release of study data was obtained before they were enrolled.

\section{Measurement of Body Weight and Blood Pressure}

Body weight and blood pressure were measured in the morning after 12 hours of fasting. Blood pressure was measured at least twice in a sitting position, and mean blood pressure was used as data.

\section{Blood Sampling}

Blood samples were collected in the morning after 12 hours of fasting. Serum was separated within 1 hour, and samples were used for measuring of fasting blood glucose (FBS), glycosylated hemoglobin 
Table 2. Comparison of change of metabolic parameters between the two groups after 12 months

\begin{tabular}{lccccc}
\hline & \multicolumn{2}{c}{ Non-acarbose group } & & \multicolumn{2}{c}{ Acarbose group } \\
\cline { 2 - 3 } \cline { 5 - 6 } & Change & $p$ & & Change & $p$ \\
\hline BMI & $-0.2 \pm 0.7$ & NS & & $-0.1 \pm 1.2$ & NS \\
BP (systolic/diastolic) & $-2 \pm 8 /-1 \pm 8$ & NS/NS & & $-2 \pm 10 /-1 \pm 16$ & NS/NS \\
FBS (mg/dL) & $-10 \pm 8$ & NS & & $-7 \pm 4$ & NS \\
Basal IRI (mU/mL) & $-0.1 \pm 0.4$ & NS & & $-0.9 \pm 0.5^{*}$ & $<0.05$ \\
HbA1c (\%) & $-0.3 \pm 0.6$ & $<0.05$ & & $-0.7 \pm 0.8^{*}$ & $<0.05$ \\
TC (mg/dL) & $2 \pm 22$ & NS & & $2 \pm 20$ & NS \\
TG (mg/dL) & $-11 \pm 43$ & NS & & $-28 \pm 58^{*}$ & $<0.05$ \\
HDL-C (mg/dL) & $1.0 \pm 4.2$ & NS & & $3.5 \pm 3.9^{*}$ & $<0.05$ \\
LDL-C (mg/dL) & $-1 \pm 13$ & NS & & $1 \pm 22$ & NS \\
\hline
\end{tabular}

Data are presented as the mean \pm SD. $p$, baseline vs after 12 months; ${ }^{*} p<0.05$ vs non-acarbosegroup.

BMI, body mass index; BP, blood pressure; FBS, fasting blood sugar; Basal IRI, basal immunoreactiveinsulin; HbA1c, glycosylated hemoglobin; TC, total cholesterol; TG, triglycerides; HDL-C, HDL-cholesterol; LDL-C, LDL-cholesterol

(HbA1c), basal immunoreactive insulin (basal IRI), total cholesterol (TC), triglyceride (TG), low-density lipoprotein cholesterol (LDL-C), high-density lipoprotein cholesterol (HDL-C), and LPL mass.

\section{HbA1c and Serum Lipids Analysis}

$\mathrm{HbA1c}$, including stable and unstable fractions, was measured by high performance liquid chromatography using the Hi-Auto A1c kit (Kyoto Daiichi Kagaku, Kyoto, Japan). Data of the stable type were used. TC and TG concentrations were measured enzymatically using a kit available from Nippon Shoji (Osaka, Japan) and an automatic analyzer (Hitachi 7150 available from Hitachi Tokyo, Japan). Low-density lipoprotein cholesterol (LDL-C) was calculated by Friedewald's method ${ }^{13)}$. HDL-C was measured by the selective inhibition method (Daiichi Pure Chemicals, Tokyo $)^{14)}$.

\section{Assay of LPL Mass}

LPL mass was measured by a sandwich enzymelinked immunosorbent assay using specific monoclonal antibody against lipoprotein lipase (Daiichi Pure Chemicals, Tokyo), as described by Kobayashi et al. ${ }^{7}$. The linearity of this assay system was observed from 5 to $400 \mathrm{ng} / \mathrm{mL}$. Within-run coefficient of variation was $2.8 \%$. Between-day coefficient of variation was $4.3 \%$.

\section{Carotid Ultrasonography}

Duplex carotid ultrasonography was performed using linear-array $7.5-\mathrm{MHz}$ transducers (EUB-525, Hitachi, Inc., Tokyo, Japan; SSA-260A, Toshiba, Inc., Tokyo, Japan ${ }^{15}{ }^{15}$. Briefly, examinations were conducted in a dark room, with the subject lying supine and the head held in the midline position or slightly tilted to either side. A careful search was performed to image the IMT of the far wall of CCA. When an optimal image was obtained, it was frozen in the end-diastolic phase to minimize variability during the cardiac cycle. IMT was measured twice bilaterally at $5 \mathrm{~mm}$ and 10 $\mathrm{mm}$ proximal to the dilatation of the CCA. Among the measurements, the highest IMT value was used. The averaged coefficient of variation in all tests was $4.8 \%$.

All measurements of CCA-IMT were performed as a part of the routine clinical work-up by the same technician, and all evaluations were performed by the same clinician. They were blinded to all clinical data of the subjects.

\section{Statistical Analysis}

Statistical analysis was performed using Student's $t$-test and paired $t$-test. In all comparisons, values of $p$ less than 0.05 were considered significant.

\section{Results}

\section{Change of Metabolic Parameters in Acarbose or Non-Acarbose Groups after 12 Months (Table 2)}

A significant decrease in HbA1c was observed in the two groups after 12 months; however, the decrease in acarbose group was significantly larger than that in the non-acarbose group. Basal IRI in the acarbose group decreased significantly, but no significant changes were observed in the non-acarbose group. TG decreased in both groups, although the decrease was significant only in the acarbose group. HDL-C increased significantly in the acarbose group, but showed no significant change in the non-acarbose group. No significant changes in BMI, BP, FBS, TC or LDL-C were observed in both groups. 
Table 3. Comparison of baseline and change of CCA-IMT and LPL mass between the two groups after 12 months

\begin{tabular}{|c|c|c|c|c|c|c|}
\hline & \multicolumn{3}{|c|}{ Non-acarbose group } & \multicolumn{3}{|c|}{ Acarbose group } \\
\hline LPL mass $(\mathrm{ng} / \mathrm{mL})$ & $49.0 \pm 17.8$ & $3.1 \pm 8.2$ & NS & $49.6 \pm 16.8$ & $11.5 \pm 9.4^{*}$ & $<0.05$ \\
\hline CCA-IMT (mm) & $0.995 \pm 0.21$ & $0.02 \pm 0.04$ & NS & $1.024 \pm 0.24$ & $-0.06 \pm 0.06^{*}$ & $<0.05$ \\
\hline
\end{tabular}

Data are presented as the mean \pm SD (LPL mass) or SE (CCA-IMT). $p$, baseline vs after 12 months; ${ }^{*} p<0.05$ vs. non-acarbose group.

CCA-IMT, common carotid artery-intima media thickness

Table 4. Comparison of baseline and change of data in subjects showing HbA1c decrease $>0.5 \%$ after 12 months

\begin{tabular}{|c|c|c|c|c|c|c|}
\hline & \multicolumn{3}{|c|}{ Non-acarbose group } & \multicolumn{3}{|c|}{ Acarbose group } \\
\hline & Baseline & Change & $p$ & Baseline & Change & $p$ \\
\hline n (male/female) & $21(7 / 14)$ & & & $31(16 / 15)$ & & \\
\hline Age (years) & $61 \pm 5$ & & & $59 \pm 4$ & & \\
\hline Morbidity duration of diabetes (years) & $11.9 \pm 7.1$ & & & $12.1 \pm 6.5$ & & \\
\hline BMI & $22.9 \pm 3.2$ & $-0.2 \pm 0.5$ & NS & $23.2 \pm 3.2$ & $-0.1 \pm 0.8$ & NS \\
\hline BP (systolic/diastolic) & $131 \pm 10 / 74 \pm 6$ & $-3 \pm 12 /-1 \pm 10$ & NS/NS & $130 \pm 9 / 75 \pm 4$ & $-2 \pm 9 /-1 \pm 12$ & NS/NS \\
\hline $\mathrm{FBS}(\mathrm{mg} / \mathrm{dL})$ & $135 \pm 15$ & $-12 \pm 6$ & NS & $132 \pm 13$ & $-9 \pm 6$ & NS \\
\hline Basal IRI $(\mu \mathrm{U} / \mathrm{mL})$ & $4.5 \pm 1.2$ & $-0.2 \pm 0.4$ & NS & $4.2 \pm 1.5$ & $-1.1 \pm 0.5^{*}$ & $<0.05$ \\
\hline HbA1c $(\%)$ & $7.5 \pm 0.8$ & $-0.8 \pm 0.3$ & $<0.05$ & $7.8 \pm 0.9$ & $-0.9 \pm 0.2$ & $<0.05$ \\
\hline $\mathrm{TC}(\mathrm{mg} / \mathrm{dL})$ & $201 \pm 34$ & $2 \pm 18$ & NS & $210 \pm 33$ & $3 \pm 22$ & NS \\
\hline $\mathrm{TG}(\mathrm{mg} / \mathrm{dL})$ & $137 \pm 75$ & $-16 \pm 57$ & NS & $140 \pm 83$ & $-31 \pm 66^{*}$ & $<0.05$ \\
\hline HDL-C (mg/dL) & $51 \pm 12$ & $1 \pm 6$ & NS & $54 \pm 11$ & $4 \pm 5^{*}$ & $<0.05$ \\
\hline LDL-C (mg/dL) & $117 \pm 31$ & $3 \pm 15$ & NS & $124 \pm 34$ & $2 \pm 25$ & NS \\
\hline LPL mass $(\mathrm{ng} / \mathrm{mL})$ & $48.6 \pm 10.2$ & $4.6 \pm 8.4$ & $<0.05$ & $47.2 \pm 11.2$ & $12.3 \pm 9.1^{*}$ & $<0.01$ \\
\hline CCA-IMT $(\mathrm{mm})$ & $0.965 \pm 0.11$ & $-0.02 \pm 0.03$ & NS & $0.962 \pm 0.08$ & $-0.08 \pm 0.04^{*}$ & $<0.05$ \\
\hline Lipid-lowering drugs (No) & $5(16 \%)$ & & & $5(24 \%)$ & & \\
\hline Blood pressure-lowering drugs (No) & $4(13 \%)$ & & & $3(14 \%)$ & & \\
\hline
\end{tabular}

Data are presented as the mean \pm SD or SE (CCA-IMT). $p$, baseline vs after 12 months; NS, not significant; ${ }^{*} p<0.05$ vs non-acarbose group; BMI, body mass index; BP, blood pressure; FBS, fasting blood sugar; Basal IRI, basal immunoreactiveinsulin; HbA1c, glycosylated hemoglobin; TC, total cholesterol; TG, triglycerides; HDL-C, HDL-cholesterol; LDL-C, LDL-cholesterol; CCA-IMT, common carotid artery-intima media thickness

\section{Baseline Data and Changes of CCA-IMT and LPL Mass in Acarbose or Non-Acarbose Group (Table 3) \\ The CCA-IMT in the acarbose group decreased significantly after 12 months of acarbose administra- tion, whereas the CCA-IMT increased slightly in the non-acarbose group. This change in CCA-IMT was significantly different between the two groups. A sig- nificant increase in LPL mass was observed after acar- bose administration for 12 months; however, no sig- nificant change in LPL mass was observed in the non- acarbose group.}

\section{Effect of Acarbose on Changes of LPL Mass or CCA-IMT Among Patients Showing Improved HbA1c (Table 4)}

Next, to clarify whether the effect of acarbose on CCA-IMT is independent of the improvement in
HbA1c, we selected patients with HbA1c who decreased by more than $0.5 \%$ after 12 months from among the two groups ( 31 patients in acarbose group, 21 patients in non-acarbose group), and compared the changes in LPL mass and CCA-IMT between these two groups. The two groups did not differ significantly in any of the baseline parameters. In the acarbose group, a significant increase in LPL mass and a significant decrease in CCA-IMT were observed after 12 months; however, no significant change of CCA-IMT was observed in the non-acarbose group, although the changes in FBS and HbA1c were almost the same in the two groups. The change in TG and increase in HDL-C were also significantly $(p<0.05)$ greater in the acarbose group than in the non-acarbose group, but no significant changes in BMI, BP, $\mathrm{TC}$ and LDL-C were observed between two groups. 
There were also no differences in the ratio of subjects using lipid-lowering or blood pressure-lowering drugs between the two groups.

\section{Discussion}

Recently, a prospective study proved that LPL mass could be an independent predictor of coronary heart disease ${ }^{16)}$. CCA-IMT is also known to be an important predictor of coronary heart disease and stroke $^{1,2)}$. Therefore, we administered acarbose to type 2 diabetes patients treated by sulfonylurea, and investigated the effect of acarbose on LPL mass and CCAIMT. The decrease of CCA-IMT and increase of LPL mass were significantly higher in the acarbose group than in the non-acarbose group. These findings suggested that acarbose had anti-atherosclerotic effects in type 2 diabetic patients treated by sufonylurea.

As for the mechanisms of LPL mass increase in the acarbose group, we speculate that acarbose administration improves insulin resistance, since LPL mass has been reported to possibly reflect insulin sensitivity ${ }^{10,17,18)}$. This speculation is supported by recent reports that postprandial hyperglycemia is associated with insulin resistance ${ }^{19,20)}$ and that acarbose improves insulin resistance ${ }^{21,22)}$. In our data, a significant decrease of basal IRI in the acarbose group also might support the improvement of insulin resistance.

Serum levels of LPL mass are reported to be related to atherosclerosis ${ }^{10,11,16)}$. We therefore examined whether the change of LPL mass influences CCAIMT. An increase of LPL mass was accompanied with a decrease of CCA-IMT. These results suggest that an increase of LPL mass could be consistent with antiatherosclerotic change and might be a clinical marker for atherosclerotic diseases. The effect of acarbose on IMT has already been reported by Hanefeld et al. ${ }^{3)}$ who showed that acarbose slows the progression of CCA-IMT in subjects with impaired glucose tolerance (IGT). Our data indicate that acarbose not only slows the progression but even decreases CCA-IMT. The reason for the difference between our data and the findings of Hanefeld et al. might be due to the difference in the subjects studied. The subjects in Hanefeld's study had IGT and the effect on CCA-IMT is largely due to the reduction of postprandial hyperglycemia by acarbose; however, our subjects were patients with type 2 diabetes mellitus, and the effect on CCA-IMT is a compound effect of the reduction of postprandial hyperglycemia by acarbose administration and also a decrease of HbA1c. In other glucose-lowering drugs, biguanide have been reported to attenuate progression of CCA-IMT ${ }^{23,24)}$, and pioglitazone is known to im- prove CCA-IMT ${ }^{25,26)}$. These effects are considered to be due to improved insulin resistance. Acarbose also might improve insulin resistance by decreasing postprandial hyperglycemia.

With respect to serum lipids, HDL-C increased significantly in the acarbose group, while the increase was not significant in the non-acarbose group. TG decreased in both groups, although the decrease was significant only in the acarbose group. Previous reports demonstrated that high levels of TG, and low levels of HDL-C and remnant lipoprotein are generally recognized in patients with low levels of preheparin LPL mass ${ }^{27,28)}$, and that an increase of preheparin LPL mass is accompanied with a decrease of TG and an increase of HDL-C ${ }^{29)}$; therefore, the improvement of serum lipid metabolism following acarbose administration observed in this study may be explained by an increase of LPL mass.

In this study, decreases of HbA1c were observed in not only the acarbose group but also the non-acarbose group. In addition to diet and exercise therapies, these decreases might be due to the effect of sulfonylurea because all subjects were treated by sulfonylurea, which might influence CCA-IMT and other parameters. The effect of only acarbose treatment should be studied in future.

In summary, our study indicated that in type 2 diabetes mellitus treated by sulfonylurea, an alphaglucosidase inhibitor, acarbose, increased LPL mass and decreased CCA-IMT. From these results, we conclude that in type 2 diabetes mellitus treated by sulfonylurea, reduction of postprandial hyperglycemia may increase LPL mass and may be useful for the prevention of macroangiopathy.

\section{References}

1) O'Leary DH, Polak JF, Kronmal RA, Manolio TA, Burke GL, and Wolfson SK Jr.: Carotid-artery intima and media thickness as a risk factor for myocardial infarction and stroke in older adults. Cardiovascular Health Study Collaborative Research Group. N Engle J Med, 1999; 340:1422

2) Bots ML, Hoes AW, Koudstaal PJ, Hofman A, and Grobbee DE: Common carotid intima-media thickness and risk of stroke and myocardial infarction: the Rotterdam Study. Circulation, 1997; 96:1432-1437

3) Hanefeld M, Koehler C, Schaper F, Fuecker K, Henkel E, and Temelkova-Kurktschiev T: Postprandial plasma glucose is an independent risk factor for increased intimamedia thickness in non-diabetic individuals. Atherosclerosis, 1999; 144:229-235

4) Bonora E, Kiechl S, Oberhollenzer F, Egger G, Bonadonna RC, Muggeo M, and Willeit J: Impaired glucose tolerance, Type II diabetes mellitus and carotid atherosclerosis: 
prospective results from the Bruneck Study. Diabetologia, 2000; 43:156-164

5) Kawamori R, Yamasaki Y, Matsushima H, Nishizawa H, Nao K, Hougaku H, Maeda H, Handa N, Matsumoto M, and Kameda T: Prevalence of carotid atherosclerosis in diabetic patients: Ultrasound high-resolution B-mode imaging on carotid arteries. Diabetes Care, 1992; 15:12901294

6) Puls W, Keup U, Krause HP, Thomas G, and Hoffmeister F: Glucosidase inhibition. A new approach to the treatment of diabetes, obesity, and hyperlipoproteinaemia. Naturwissenschaften, 1977; 64:536-537

7) Kobayashi J, Hashimoto H, Fukamachi I, Tashiro K, Shirai K, Saito Y, and Yoshida S: Lipoprotein lipase mass and activity in severe hypertriglyceridemia. Clin Chem Acta, 1993; 216:113-123

8) Tornvall P, Olivecrona G, Karpe F, Hamsten A, and Olivecrona T: Lipoprotein lipase mass and activity in plasma and there increase after heparin are separate parameters with different relations to plasma lipoproteins. Arterioscler Thromb Vasc Biol, 1995; 15:1086-1093

9) Miyashita Y, Shirai K, Itoh Y, Sasaki H, Totsuka M, Murano T, and Watanabe H: Low lipoprotein lipase mass in preheparin serum of type 2 diabetes mellitus patients and its recovery with insulin therapy. Diabetes Res Clin Pract, 2002; 56:181-187

10) Shirai K, Itoh I, Sasaki H, Totsuka M, Murano T, Watanabe $\mathrm{H}$, and Miyashita $\mathrm{Y}$ : The effect of insulin sensitizer, troglitazone, on lipoprotein lipase mass in preheparin serum. Diabetes Res Clin Pract, 1999; 46:35-41

11) Hitsumoto $T$, Ohsawa $H$, Uchi $T$, Noike $H$, Kanai $M$, Yoshinuma M, Miyashita Y, Watanabe H, and Shirai K: Preheparin serum lipoprotein lipase mass is negatively related to coronary atherosclerosis. Atherosclerosis, 2000; 153:391-396

12) Hitsumoto T, Yoshinaga K, Aoyagi K, Sakurai T, Kanai M, Uchi T, Noike H, Ohsawa H, Watanabe H, and Shirai K: Association between preheparin serum lipoprotein lipase mass and acute myocardial infarction in Japanese men. J Atheroscler Thromb, 2002; 9:163-169

13) Friedewald WT, Levy RI, and Fredrickson DS: Estimation of the concentration of low-density lipoprotein cholesterol in plasma, without use of the preparative ultracentrifuge. Clin Chem, 1972; 18:499-502

14) Shirai K, Nema T, Hiroh Y, Itoh Y, Miyashita Y, and Watanabe $\mathrm{H}$ : Clinical efficacy of the direct assay method using polymers for serum high density lipoprotein cholesterol. J Clin Lab Anal, 1997; 11:82-86

15) Nagai Y, Kitagawa K, Yamagami H, Kondo K, Hougaku $\mathrm{H}$, Hori M, and Matsumoto M: Carorid artery intimamedia thickness and plaque score for the risk assessment of stroke subtypes. Ultrasound Med Biol, 2002; 28:12391243

16) Rip J, Nierman MC, Wareham NJ, Luben R, Bingham SA, Day NE, van Miert JN, Hutten BA, Kastelein JJ, Kuivenhoven JA, Khaw KT, and Boekholdt SM: Serum lipoprotein lipase concentration and risk for future coronary artery disease: the EPIC-Norfolk prospective population study. Arterioscler Thromb Vasc Biol, 2006; 26:637642
17) Hanyu $O$, Miida $T$, Obayashi K, Ikarashi $T$, Soda $S$, Kaneko S, Hirayama S, Suzuki K, Nakamura Y, Yamatani $\mathrm{K}$, and Aizawa Y: Lipoprotein lipase (LPL) mass in preheparin serum reflects insulin sensitivity. Atherosclerosis, 2004; 174:385-390

18) Kobayashi J, Kusunoki M, Murase Y, Kawashiri M, Higashikata T, Miwa K, Katsuda S, Takata M, Asano A, Nohara A, Inazu A, and Mabuchi H: Relationship of lipoprotein lipase and hepatic triacylglycerol lipase activity to serum adiponectin levels in Japanese hyperlipidemic men. Horm Metab Res, 2005; 37:505-509

19) Shiba T: Improvement of insulin resistance by a new insulin secretagogue, nateglinide--analysis based on the homeostasis model. Diabetes Res Clin Pract, 2003; 62:8794

20) Annuzzi G, De Natale C, Iovine C, Patti L, Di Marino L, Coppola S, Del Prato S, Riccardi G, and Rivellese AA: Insulin resistance is independently associated with postprandial alterations of triglyceride-rich lipoproteins in type 2 diabetes mellitus. Arterioscler Thromb Vasc Biol, 2004; 24:2397-2402

21) Yamamoto M and Otsuki M: Effect of inhibition of alphaglucosidase on age-related glucose intolerance and pancreatic atrophy in rats. Metabolism, 2006; 55:533-540

22) Nakamura $K$, Yamagishi $S$, Matsui $T$, and Inoue $H$ : Acarbose, an alpha-glucosidase inhibitor, improves insulin resistance in fructose-fed rats. Drugs Exp Clin Res, 2005; 31:155-159

23) Matsumoto K, Sera Y, Abe Y, Tominaga T, Yeki Y, and Miyake S: Metformin attenuates progression of carotid arterial wall thickness in patients with type 2 diabetes. Diabetes Res Clin Pract, 2004; 64:225-228

24) Katakami N, Yamasaki Y, Hayaishi-Okano R, Ohtoshi K, Kaneto H, Matsuhisa M, Kosugi K, and Hori M: Metformin or gliclazide, rather than glibenclamide, attenuate progression of carorid intima-media thickness in subjects with type 2 diabetes. Diabetologia, 2004; 47:1906-1913

25) Koshiyama H, Shimono D, Kuwamura N, Minamikawa J, and Nakamura Y: Rapid communication: inhibitory effect of pioglitazone on carotid arterial wall thickness in type 2 diabetes. J Clin Endocrinol Metab, 2001; 86:3452-3456

26) Langenfeld MR, Forst T, Hohberg C, Kann P, Lübben G, Konrad T, Füllert SD, Sachara C, and Pfützner A: Pioglitazone decreases carotid intima-media thickness independently of glycemic control in patients with type 2 diabetes mellitus: results from a controlled randomized study. Circulation, 2005; 111:2525-2531

27) Watanabe $H$, Miyashita $Y$, Murano T, Hiroh $Y$, Itoh $Y$, and Shirai K: Preheparin serum lipoprotein lipase mass level: the effects of age, gender, and types of hyperlipidemias. Atherosclerosis, 1999; 145:45-50

28) Hirano T, Nishioka F, and Murakami T: Measurement of the serum lipoprotein lipase concentration is useful for studying triglyceride metabolism: Comparison with postheparin plasma. Metabolism, 2004; 53:526-531

29) Totsuka M, Miyashita Y, Ito Y, Watanabe H, Murano T, and Shirai K: Enhancement of preheparin serum lipoprotein lipase mass by bezafibrate administration. Atherosclerosis, 2000; 153:175-179 